\title{
Identification of Substandard and Falsified Medicines: Influence of Different Tolerance Limits and Use of Authenticity Inquiries
}

\author{
Cathrin Hauk, Nhomsai Hagen, and Lutz Heide* \\ Pharmaceutical Institute, Eberhard Karls University Tuebingen, Tuebingen, Germany
}

\begin{abstract}
Substandard and falsified medicines have severe public health and socioeconomic effects, especially in low- and middle-income countries. The WHO has emphasized the need for reliable estimates of the prevalence of such medicines to efficiently respond to this problem. In the present study, we used 601 medicine samples collected in Cameroon, the DR Congo, and Malawi to assess the rates of substandard and falsified medicines based on different criteria. Based on the specifications of the U.S. Pharmacopoeia for the amount of the active pharmaceutical ingredients, the rate of out-of-specification medicines was $9.3 \%$. By contrast, this rate ranged from $3.3 \%$ up to $35.0 \%$ if the tolerance limits of other pharmacopoeias or recently published medicine quality studies were used. This shows an urgent need for harmonization. Principal methods to assess the rate of falsified medicines are packaging analysis, chemical analysis, and authenticity inquiries. In the present study, we carried out an authenticity inquiry for the aforementioned medicine samples, contacting 126 manufacturers and 42 distributors. Response rates were higher for samples stated to be manufactured in Asia (52.4\%) or Europe (53.8\%) than for samples manufactured in Africa $(27.4 \% ; P<0.001)$. One sample had been identified as falsified by packaging analysis by the local researchers and two additional ones by chemical analysis. Notably, seven additional falsified samples were identified by the authenticity inquiries. The total rate of falsified medicines resulted as $1.7 \%$. Considerations are discussed for assessing the rates of "substandard" and "falsified" medicines in future medicine quality studies.
\end{abstract}

\section{INTRODUCTION}

The spread of substandard and falsified medicines (SF medicines) has been described as a pandemic, ${ }^{1}$ and its extremely severe public health and socioeconomic effects, especially in low- and middle-income countries (LMICs), are well documented. ${ }^{2}$ To respond efficiently to this problem, reliable estimates of the prevalence of SF medicines, for different geographical regions, different parts of the supply chain, and different types of medicines, are of essential importance. However, the current estimates of the prevalence rate of SF medicines in LMICs differ considerably. From a systematic review of 100 medicine quality studies in the years 2007-2016 (total 48,218 samples), an authoritative review by the $\mathrm{WHO}^{2}$ estimated the combined prevalence of SF medicines as $10.5 \%$ on average. Similarly, a systematic review of 96 studies up to the year 2017 (total 67,839 samples) by Ozawa et al. ${ }^{3}$ reported a prevalence of $13.6 \%$. By contrast, a systematic review of 15 studies up to January 2013 (total 3,931 samples) by Almuazini et al. ${ }^{4}$ reported a prevalence of $28.5 \%$, and a consecutive review by McManus and Naughton, ${ }^{5}$ using the same methodology as Almuazini et al. ${ }^{4}$ and including 33 studies published from January 2013 until December 2018 (total 19,921 samples), reported a prevalence of $25 \%$. Notably, none of these four reviews was able to state the rate of falsified medicines separately from the rate of substandard medicines because the heterogeneity of the methodologies and definitions used in the reviewed studies did not allow to aggregate prevalence data for falsified medicines.

Even larger discrepancies can be observed between earlier reviews or between individual medicine quality studies. A review in Lancet Infectious Diseases by Nayyar et al. ${ }^{6}$ reported that $20 \%$ out of 2,297 antimalarials collected in 21 countries of subSaharan Africa were falsified. In contrast, Kaur et al. ${ }^{7}$ stated that

*Address correspondence to Lutz Heide, Pharmaceutical Institute, Eberhard Karls University Tuebingen, Auf der Morgenstelle 8, Tuebingen 72076, Germany. E-mail: heide@uni-tuebingen.de only $1 \%$ of 10,079 samples of antimalarials, collected mostly in countries of sub-Saharan Africa, were falsified. Equally striking, Khuluza et al. $^{8}$ reported the prevalence of substandard antimalarials and antibiotics in Malawi to be $11 \%$, while Chikowe et al. ${ }^{9}$ reported for the same country and a similar time period a prevalence of $88 \%$ substandard antimalarials.

With such divergent estimates, an effective response to the problem of SF medicines becomes very difficult. The aforementioned review by the $\mathrm{WHO}^{2}$ therefore emphasized the urgent need for additional, reliable prevalence estimates generated with appropriate and comparable methodologies.

After many years of controversy, the World Health Assembly of 2017 has adopted universally accepted definitions of "substandard," "falsified," and "unregistered/unlicensed" medical products, ${ }^{2,10}$ and has thereby laid an indispensable foundation for the generation of reliable and comparable prevalence estimates:

1. "Substandard medical products: Also called 'out of specification'; these are authorized medical products that fail to meet either their quality standards or their specifications, or both."

2. "Falsified medical products: Medical products that deliberately/fraudulently misrepresent their identity, composition, or source."

According to the WHO, these definitions are mutually exclusive, so a sample can either be classified as "substandard" or as "falsified". 10

The previously cited wording "Substandard medical products: Also called 'out of specification'..." appears to use the term "substandard" synonymous with the term "out of specification." This may sometimes cause confusion because, according to the U.S. Food and Drug Administration, out-ofspecification (OOS) results include all test results that fall outside the relevant specifications or acceptance criteria, ${ }^{11}$ irrespective of the absence or presence of deliberate intent as the reason for noncompliance of the investigated product. 
Notably, the cited WHO document adds a footnote to its definition of substandard medical products: "When the authorized manufacturer deliberately fails to meet these quality standards or specifications due to misrepresentation of identity, composition, or source, then the medical product should be considered 'falsified'."10,12

In this study, we use the term "out of specification" for all medicines for which test results fall outside the relevant specifications. We use the term "substandard" for those medicines for which test results fall outside the relevant specifications AND for which no evidence is available that they deliberately/fraudulently misrepresent their identity, composition, or source. Substandard quality may result from shortcomings in the production process, and/or from degradation after the product has been manufactured (see also Discussion section).

Guidelines for the conduct and the reporting of medicine quality studies have been published by Newton et al. ${ }^{13}$ and by the $\mathrm{WHO},{ }^{14}$ and as documented by McManus and Naughton, ${ }^{5}$ this led to a noticeable improvement of the quality of such studies in the recent years.

The present article aims to contribute to a further improvement of the methodology and reporting of medicine quality studies. We focus on two aspects mentioned in the WHO re$v^{2} w^{2}$ : first, different authors and even different pharmacopoeias use different thresholds to distinguish between "in-specification" and "out-of-specification" medicines. Using the analytical data of 601 medicine samples which were recently collected in Cameroon, the DR Congo, and Malawi, and analyzed in our laboratory, ${ }^{15,16}$ we here determined the effect of the use of different thresholds on the resulting prevalence rates of OOS medicines. This exercise resulted in strikingly different rates. Second, we conducted an authenticity inquiry, contacting the manufacturers and distributors of the aforementioned medicine samples. Unexpectedly, this increased the number of falsified medicines from three (which had previously been identified by packaging and chemical analyses) to a new total of 10 falsified samples. We report details of the procedures used and the response rates obtained in this authenticity inquiry. Finally, we present considerations for assessing the rates of "substandard" and "falsified" medicines in future medicine quality studies.

\section{METHODS}

Ethical approval. Ethical approvals and permissions to conduct the medicine quality studies were obtained from the responsible authorities in Cameroon, the DR Congo, and Malawi. ${ }^{15,16}$

Study design and included medicines. The studies were designed according to the MEDQUARG guidelines ${ }^{13}$ and the WHO guidelines on the conduct of surveys of the quality of medicines. ${ }^{14}$ In Cameroon and the DR Congo, seven antibiotics and six medicines against noncommunicable diseases were included (see Figure 1), with all of them representing solid oral formulations in dosages for adults. In Malawi, oxytocin injections and misoprostol tables were sampled. All included medicines were contained in the essential medicines lists of the respective countries. ${ }^{15,16}$

Sample collection. Sample collection took place between August 2017 and November 2018. Details of the collection process have been described previously. ${ }^{15,16}$ For the purpose of these studies, a "sample" was defined as medical product of a specific brand and batch, sampled at the same time and same place.

Chemical analysis, and classification of medicines as OOS using different tolerance limits. Solid oral formulations were analyzed for identity, assay (= content of the active pharmaceutical ingredient [API]) and dissolution at the Pharmaceutical Institute of Tuebingen University according to the monographs of the U.S. Pharmacopoeia (USP) 41, except for misoprostol tablets which were analyzed according to the International Pharmacopoeia (Ph. Int.), Seventh Edition (2017) because no USP monograph was available for this product. Oxytocin injections were analyzed for identity, assay, and $\mathrm{pH}$ according to USP $40 .^{15,16}$

To allow a comparison of the quality of samples from different manufacturers, $\mathrm{WHO}$ guidelines ${ }^{14}$ recommend that in one medicine quality study, all samples which contain the same API in the same dosage form should be tested using the same methods and specifications, irrespective of possibly different methods and specifications used by the manufacturers for the registration of their products. The specifications of USP 42 (2019), Ph. Int. Ninth Edition (2019) and BP (2020) were used to calculate the effect of different thresholds on the rate of OOS medicines in this study. Tolerance limits of USP 40,41 , and 42 are identical for the investigated medicines, and also tolerance limits of the seventh and the ninth edition of $\mathrm{Ph}$. Int. are identical for misoprostol.

Packaging analysis and authenticity inquiries. The brand name, batch number, manufacturing date, expiry date, name of manufacturer and distributor, international nonproprietary names of the APIs, strength, dosage form, and package size were recorded. The information stated on the labels and on the package insert was examined for the presence of irregularities or possible signs of falsification, such as spelling mistakes.

Requests for authentication were sent to all stated manufacturers and distributors, irrespective of the results of packaging analysis and chemical analysis. Websites of these companies were identified from the labeling or from the package inserts as well as from the Internet using the search engines Google (Mountain View, CA), Microsoft Bing (Redmond, WA), and Baidu (Beijing, China). Manufacturers and distributors were contacted using the e-mail addresses stated on the packaging or on their respective website if possible. If no website could be found, or if no e-mail addresses were stated there, e-mail addresses were searched using the aforementioned search engines. Inquiries were sent by post, if no e-mail address could be found or obviously none of our e-mail inquiries reached the recipient, for example, if an error message was received in response. For 225 of these samples, not only a manufacturer but also a distributor was stated on the packaging, and both were contacted. Two samples only stated a distributor, but not the manufacturer. Six distributors are also contained in the list of manufacturers (Supplemental Table S3), because they acted as distributors for certain products, and as manufacturers for others. As stated in the footnotes of Supplemental Tables S3 and S4, our Internet searches revealed that a few manufacturers/distributors stated slightly different versions/abbreviations of their company's name on the labels of different collected medicines, however, with these different versions/abbreviations leading us to the same website, e-mail, or postal address. Despite the slightly different versions/abbreviations of the name, the respective manufacturers/distributors were considered as a single company in such cases. 
United States Pharmacopeia (USP 42) International Pharmacopoeia (Ph. Int. 2019) British Pharmacopoeia (BP 2020)

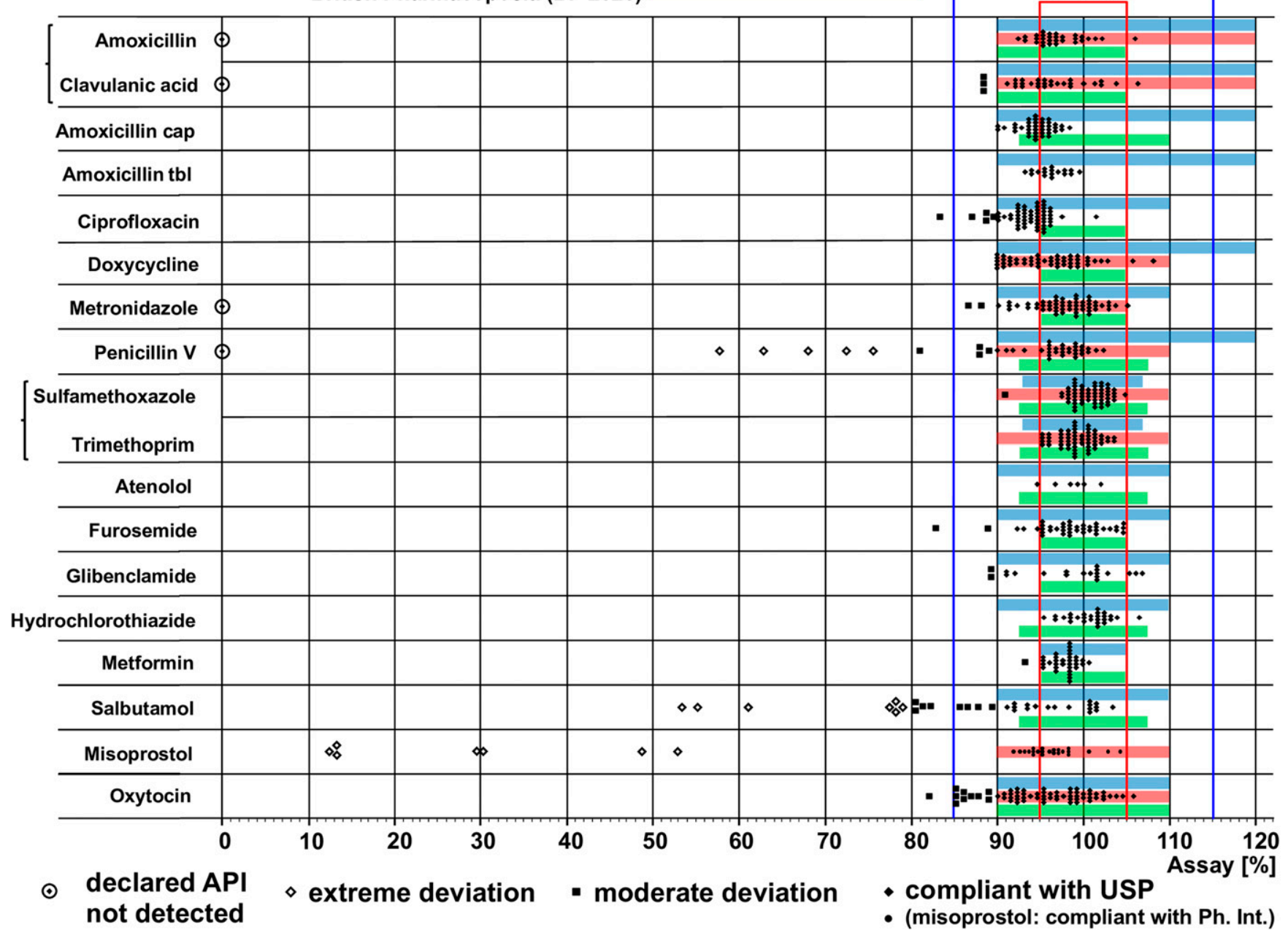

FIGURE 1. Assay results for 601 medicine samples collected in Cameroon, DR Congo, and Malawi in comparison to the tolerance limits of U.S. Pharmacopoeia (USP) 42, Ph. Int. Ninth Edition and BP 2020, and to arbitrary tolerance limits used in previous medicine quality studies (85-115\% ${ }^{7,28}$ and $95-105 \%{ }^{17}$ ). Except for oxytocin injections, all medicines in this figure represent solid oral formulations (tablets or capsules; see Supplemental Table S1). All three pharmacopoeias contain separate monographs for doxycycline tablets and doxycycline capsules, but the stated tolerance limits are identical for both formulations. The USP contains separate monographs for amoxicillin tablets and amoxicillin capsules, but BP contains only a monograph for amoxicillin capsules. Therefore, amoxicillin capsules and tablets are listed separately here. This figure appears in color at www.ajtmh.org.

The first authenticity inquiries were sent to the manufacturers and distributors of oxytocin and misoprostol in December 2019, and to the manufacturers and distributors of the other medicines in March 2020. If there was no response within six weeks after the first inquiry, an e-mail reminder was sent to the same e-mail addresses which had been contacted previously, and in addition, other e-mail addresses were searched on the Internet and contacted. If the website of the manufacturer/distributor or another trading platform offered the option to write a message to that company, an inquiry was sent in this way. If, despite these reminders, no response was received within 3 months after the first inquiry, the respective company was classified as a nonresponder. Responses obtained within 3 months after the first inquiry were evaluated.

The inquiry letter is shown in Supplemental Figure S1. It provided the brand name, stated manufacturer and distributor, dosage form and strength, batch number, manufacturing and expiry dates, as well as photos of the packaging and of the dosage units (i.e., tablets, capsules, or ampoules). The letter stated the following questions: "1. Do the batch number and the manufacturing and expiry dates correspond to your records? If yes, do the product and packaging shown on the provided photographs appear to be genuine? 2. To your knowledge, has the batch been distributed in (country of collection of the respective sample)?" The letter also mentioned that a publication of the results of this study was intended, and that the cooperation of that company would be mentioned therein. If the manufacturers/distributors requested, further details were provided on the process and objectives of this study. However, the results of the chemical analysis of the respective samples were not stated before the manufacturer/ distributor had responded regarding the authenticity of the samples.

Statistical evaluations. Statistical evaluations were performed using JMP 15 (SAS GmbH, Heidelberg, Germany). 
Significance of differences in the response rate of manufacturer and distributors between different groups was calculated using Pearson's chi-square test.

\section{RESULTS}

Determination of the rate of oos medicines using different tolerance limits. In our recent medicine quality studies in Cameroon, the DR Congo, and Malawi, ${ }^{15,16} 601$ samples of 15 different medicines had been collected. Thirteen of these medicines contained a single API each, and two were fixeddose combinations of two APIs. Of these 15 medicines, 14 were covered by monographs in the current editions of USP and BP, and 7 by a monograph in Ph. Int.

As illustrated in Figure 1, the tolerance limits given in the three pharmacopoeias for assay testing (i.e., for the content of the API) are markedly different, ranging from $95-105 \%$ to 90-120\% of the declared content. The numerical values for these tolerance limits are listed in Supplemental Table S1. Figure 1 also shows the arbitrary tolerance limits of $85-115 \%$ chosen by Kaur et al. ${ }^{7}$ for a large study of antimalarials in various (mostly African) countries published in 2016, and of 95-105\% chosen by Antignac et al. ${ }^{17}$ for a large study of cardiac medicines in seven African countries published in 2017. Figure 1 also depicts the individual assay results obtained for each of the 601 samples collected in our studies. For a few of the investigated medicines, such as co-trimoxazole (sulfamethoxazole and trimethoprim) or metformin tablets, the percentage of OOS samples is similar, irrespective of which tolerance limits are applied. For most medicines, however, the choice of the tolerance limits has a huge influence on the resulting number of OOS medicines. For example, of the 57 collected samples of ciprofloxacin tablets, five (8.8\%) were OOS using the tolerance limits of USP, whereas 43 (75.4\%) were OOS using the tolerance limits of BP. Supplemental Table S2 shows the rate of OOS medicines for each type of medicine, using the different mentioned tolerance limits.

The overall rates of OOS samples for all types of medicines together, calculated using the different mentioned tolerance limits, are depicted in Figure 2 and in the Supplemental Figure S2. For exact mathematical comparison, Figure 2 includes only the results of those 300 samples for which monographs exist in all three mentioned pharmacopoeias. The rate of OOS
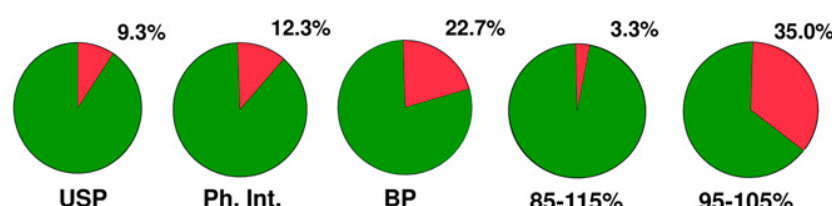

FiguRE 2. Influence of different tolerance limits on the rate of out-ofspecification medicines (assay results) calculated for medicine samples collected in Cameroon, DR Congo, and Malawi (OOS: red; in-specification: green). The rates of out-of-specification medicines were calculated using the specifications of USP 42, Ph. Int. Ninth Edition and BP 2020, and the arbitrary tolerance limits $85-115 \%{ }^{7,28}$ and $95-105 \%,{ }^{17}$ respectively. For this figure, only the assay results of the six investigated medicines for which monographs exist in all three pharmacopoeias were compared ( $n=300$ samples). Supplemental Figure 22 shows a similar comparison including all medicines investigated in the three countries ( $n=601$ samples). This figure appears in color at www.ajtmh.org. samples resulting from the use of USP and $\mathrm{Ph}$. Int. specifications is $9.3 \%$ and $12.3 \%$, respectively, but it is markedly higher $(22.7 \%)$ when the specifications of BP are used. Using the arbitrary limits of $85-115 \%$ chosen by Kaur et al., ${ }^{7}$ only $3.3 \%$ of the samples result as OOS. In sharp contrast, $35.0 \%$ (i.e., more than 10 times as many!) are OOS if the limits of 95-105\% chosen by Antignac et al. ${ }^{17}$ are used. Supplemental Figure S2 shows the same comparison including all samples collected in Cameroon, the DR Congo, and Malawi. The results are similar to those depicted in Figure 2.

Different pharmacopoeias specify different methods to be used in quality analysis. For assay testing, results obtained with these different methods are expected to be similar at least in most cases. ${ }^{18}$ By contrast, for the dissolution of an API (i.e., in vitro drug release from a solid oral formulation), different pharmacopoeial methods use different dissolution media and are well known to yield quite different results, for example, in the case of rifampicin. ${ }^{19-22}$ Therefore, we did not attempt to evaluate the dissolution results which we obtained in our previous studies ${ }^{15,16}$ with the tolerance limits of different pharmacopoeias.

Assessment of the rate of falsified medicines through packaging analysis. According to $\mathrm{WHO}$ guidelines, ${ }^{14}$ the packaging of each collected sample, its labeling, and its package leaflet should be inspected visually for any signs of being a substandard or falsified product. Checklists for this purpose have been published ${ }^{23,24}$ and may allow the identification of suspicious medicine samples by frontline health workers even before any chemical analysis is performed. Among the 601 samples collected in our studies, only one was readily identified as probably falsified by our local cooperation partners in Cameroon based on packaging analysis. It was stated to represent penicillin $\mathrm{V}$ tablets, but contained gross spelling errors in the labeling. ${ }^{15,25}$ Further investigation showed that the stated manufacturer ("Oxford Pharma Co. Ltd. Belgium") was a non-existing company, and subsequent chemical analysis showed that the tablets contained no penicillin V but a small amount of paracetamol. ${ }^{15}$ Based on these findings, the WHO classified this product as "falsified" in their Medical Product Alert N ${ }^{\circ} 4 / 2017 .{ }^{25}$

Also, in the leaflet of an oxytocin brand produced in China, a high number of spelling errors were found. However, spelling errors in English language are not uncommon for products from some manufacturers in non-English-speaking countries, and were not considered to indicate a falsified medicine in this case. Indeed, in the authenticity inquiry (see in the following text), the distributor confirmed the authenticity of the samples of this brand. However, of the 38 samples of this brand, six samples were substandard because they contained only 85.2-86.8\% of the declared amount of the API (USP tolerance limit: $90-110 \%) .{ }^{16}$ Further testing also revealed shortcomings in the stability of this product. ${ }^{26}$

Assessment of the rate of falsified medicines through chemical analysis. In addition to the falsified penicillin $\mathrm{V}$ tablets mentioned earlier, chemical analysis of the 601 samples identified two further samples which did not contain detectable amounts of the stated API. ${ }^{15}$ One sample was labeled as "Augmentin ${ }^{\circledR}$ GlaxoSmithKline - SmithKline Beecham Pharmaceuticals (Worthing, West Sussex, UK), (amoxicillin 500 mg/ clavulanic acid $125 \mathrm{mg}$ tablets)," but contained no detectable amounts of the active ingredients. ${ }^{15,27}$ The stated manufacturer confirmed that this sample was falsified, and it was subsequently 
reported as "falsified" by the WHO in their Medical Product Alert $\mathrm{N}^{\circ} 2 / 2018 .{ }^{27}$ Another sample, sold by an informal vendor from an opened plastic container, was labeled as "Metronyl ${ }^{\circledR}$ Metronidazole Tablets B.P., Mac's Pharmaceutical Ltd. (Nairobi, Kenya)," but contained a small amount of the prodrug metronidazole benzoate instead of metronidazole. The stated manufacturer was informed, but no answer was received. $^{15}$

Assessment of the rate of falsified medicines through authenticity inquiries. Manufacturers and distributors were asked to confirm the authenticity of the medicine sample, and to state whether the sample had been distributed to the country where it had been collected in our studies (see Methods section). As shown in Figure 3, 582 samples were included into the authenticity inquiry. These represented 272 different brands (427 different batches). In 12 cases, the manufacturer or distributor could not be contacted online and was therefore only contacted by regular mail. Because of an interruption of the postal services to several African countries caused by the corona pandemic, three distributors could not be reached by regular mail, but for each of the concerned samples, the manufacturer could be contacted. Supplemental Tables S3 and S4 list all 126 manufacturers and 42 distributors that have been contacted, with the information whether or not they responded to our inquiry. An answer with a statement on authenticity was received for $288(49.5 \%)$ of the samples. For 281 samples, the authenticity was confirmed by the manufacturer and/or the distributor, whereas seven samples were stated to be falsified. For 50 of the 288 samples (17.4\%), answers were received both from the manufacturer and from the distributor; in all these cases, the information from both sources regarding the authenticity of the sample was in agreement (see Supplemental Table S5).

Table 1 summarizes the response rates received from different geographical regions. For samples stated to be manufactured in Asia, or in Europe and the Americas, response rates were slightly greater than $50 \%$ and therefore higher than those for samples stated to be manufactured in Africa (27.4\%; $P<0.001)$. India and China were the most frequent countries of origin for the medicines collected in our studies, and overall response rates for samples manufactured in both countries were similar (53.7\% and $50.3 \%$, respectively). It should be noted, however, that for the samples manufactured in China, responses were in most cases only received from the distributors which were predominantly located in Africa and

\section{6 samples collected in DR Congo and Cameroon 95 samples collected in Malawi}

\begin{tabular}{|l|l|}
\hline & 3 samples already identified as falsified by chemical investigation \\
\hline 582 samples included in authenticity enquiry: \\
$>355$ samples: only manufacturer stated on packaging
\end{tabular}

FIGURE 3. Flowchart of the identification of falsified medicines by authenticity inquiries to manufacturers and distributors. Five extremely substandard samples of misoprosto ${ }^{16}$ were excluded, because upon our alert letter, the WHO had suggested that they contact the manufacturers and distributors. 
TABLE 1

Response rates obtained in the authenticity inquiry, listed by geographic region

\begin{tabular}{|c|c|c|c|c|c|c|}
\hline & \multicolumn{2}{|c|}{ Samples } & \multicolumn{2}{|r|}{ Manufacturers } & \multicolumn{2}{|r|}{ Distributors* } \\
\hline & $\begin{array}{l}\text { Number of samples } \\
\text { manufactured in this region }\end{array}$ & $\begin{array}{l}\text { Number (\%) of samples with } \\
\text { authenticity statement }\end{array}$ & $\begin{array}{l}\text { Number } \\
\text { contacted }\end{array}$ & $\begin{array}{l}\text { Number (\%) of responses with } \\
\text { authenticity statement }\end{array}$ & $\begin{array}{l}\text { Number } \\
\text { contacted }\end{array}$ & $\begin{array}{l}\text { Number (\%) of responses with } \\
\text { authenticity statement }\end{array}$ \\
\hline Asia (total) & 420 & 220 (52.4) & 83 & 30 (36.1) & 9 & $4(44.4)$ \\
\hline India & 257 & $138(53.7)$ & 58 & 28 (48.3) & 7 & $3(42.9)$ \\
\hline $\begin{array}{l}\text { China and } \\
\text { Hong Kong }\end{array}$ & 161 & 81 (50.3) & 24 & $1(4.2)$ & 1 & 0 \\
\hline $\begin{array}{l}\text { Europe and } \\
\text { Americas }\end{array}$ & 96 & $51(53.1)$ & 24 & 13 (54.2) & 16 & 7 (43.8) \\
\hline Africa & 62 & 17 (27.4) & 19 & 6 (31.6) & $17 \dagger$ & 5 (29.4) \\
\hline Total & $582 \ddagger$ & 288 (49.5) & 126 & 49 (38.9) & $42 \dagger$ & 16 (38.1) \\
\hline
\end{tabular}

*Distributors were often located in another region than the manufacturers of the respective samples.
† There were 20 distributors from the African continent in this study, but for three of them, representing 14 samples, no e-mail address or contact website was found, and they could not be contacted by regular mail as postal services were interrupted because of the COVID-19 pandemic. Therefore, only 42 of the 45 distributors represented in this study could be contacted.

$\ddagger$ Five hundred eighty-two samples were included into the authenticity inquiry, but for four samples, the country of origin was unknown; for three samples from Cinpharm, it was not stated whether they had been manufactured by Cinpharm in Cameroon or by their partner Cipla Ltd. in India; and for one sample, the available packaging only stated the distributor but not the manufacturer.

Europe, whereas of the 24 Chinese manufacturers, only a single one responded to our inquiry (Table 1).

We also investigated whether the response rates were related to the quality of the samples. For the 496 samples which had been found to comply to the assay and (if applicable) dissolution specifications of the USP, or of Ph. Int. in case of misoprostol samples, the response rate obtained was $51.2 \%$. For the 86 samples which did not comply with pharmacopeial specifications, the response rate was $39.5 \%$. This difference just reached statistical significance $(P=0.0456)$.

Including all the 49 manufacturers and 16 distributors who responded to our inquiry, the median time between the first inquiry and the receipt of a response with an authenticity statement was 16 days (range 0-84 days). Thirty eight (58.5\%) of these manufacturers and distributors responded already to the first inquiry, whereas in 27 cases (41.5\%), a reminder was required (see Methods sections). Notably, the median time between reminder and receipt of a response was only 2 days (range 0-54 days). Supplemental Tables S3 and S4 list all manufacturers and distributors who were contacted, with the respective number of days between first contact and response, and (if applicable) between reminder and response.

Of the 281 samples for which authenticity was confirmed, for 165 samples (58.7\%), the manufacturer and/or distributor additionally confirmed that the sample had been distributed to the country from where it had been collected in our studies. For 45 samples (16.0\%), it was denied that the sample had been distributed to the respective country, suggesting that these samples had been further traded to other countries by the first buyer. For 71 samples (25.3\%), this question was not answered.

Falsified medicines identified by authenticity inquiries. Three falsified samples had been identified by packaging and chemical analyses (see aforementioned). ${ }^{15}$ Seven further samples (representing four different brands) were only identified as falsifications through the authenticity inquiries. Notably, all seven samples passed chemical analysis for assay and dissolution testing according to the USP specifications. These falsified medicines are shown in Figure 4A-D. One of them (Figure 4A) had been collected in Cameroon from an informal vendor and was labeled as "Amoxicillin 500 mg + Clavulanic acid 125 mg BP" tablets, stated manufacturer: Medopharm Pvt. Ltd. (Guduvanchery, India), stated distributor: IDA Foundation (Amsterdam, The Netherlands). According to the information received from the stated manufacturer and distributor, the batch number did not match products of their companies, the stated shelf life (3 years) was different from that of the genuine product ( 2 years), and also the length of the blister pack $(220 \mathrm{~mm})$ did not match that of the genuine product $(195 \mathrm{~mm})$ (see Supplemental Figure S3). Also, some further details in the labeling (e.g., postal code in the address of the stated manufacturer) were stated to be incorrect.

Another sample (Figure 4B) had also been collected in Cameroon from an informal vendor, in another region of the country. It was labeled as "CO-TRIMOXAZOLE (sulfamethoxazole \& trimethoprim)" tablets, batch number 13617, stated manufacturer: Sprukfield (Lomé, Togo). Notably, three further co-trimoxazole samples labeled as manufactured by Sprukfield had been collected in our study from church and government health facilities in Cameroon, ${ }^{15}$ in these cases showing the batch numbers AT15001 (two samples) and AT15007 (one sample). On our inquiry, Sprukfield stated that the sample with the batch number 13617 had not been produced by them and was falsified, whereas the three other samples were confirmed to be genuine. The falsified product showed several differences in comparison to the genuine ones (Figure 4B): the falsified product was packaged in a slightly shorter blister pack, the batch number and expiry date were printed rather than being embossed on the blister, and the artwork of the Sprukfield logo and the green color used for blister labeling were different from those of the genuine products. Also, the embossing of the tablets was different (Figure 4B).

Four samples of "Furosemide 40 mg BP" tablets, stated manufacturer: Micro Labs Ltd. (Hosur, India), stated distributor: IDA Foundation, had been collected in church health facilities in four different regions of Cameroon. Packaging and labeling of all of them were identical, and all carried the same batch number FRIH0077. However, two of them showed as manufacturing and expiry dates "Dec. 2015/Nov. 2019" (i.e., a shelf life of 4 years), whereas two others showed "Dec. 2012/Nov. 2018" (i.e., an unusual long shelf life of 6 years). Figure $4 \mathrm{C}$ depicts one of the latter samples. The manufacturer and the distributor confirmed that a corresponding product with the same artwork had been supplied to Lagos, Nigeria, with the named batch number and a manufacturing date of December 2012, but that the expiry date of that product had been November 2015 (corresponding to a shelf life of 3 years). The stated manufacturer and distributor therefore stated that the four samples we had collected were falsified. As visible in Figure 4C, the part of the label where batch number, manufacturing, and expiry dates were printed appears slightly darker than the rest of the label, suggesting that this information may have been modified. 
A
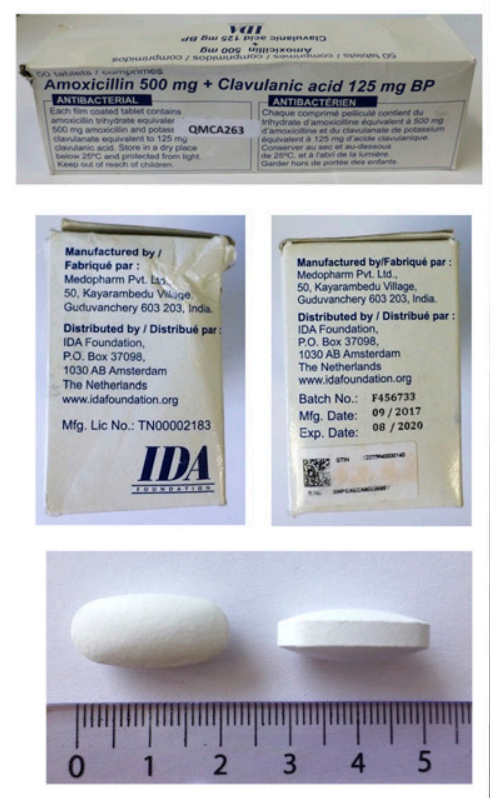

B
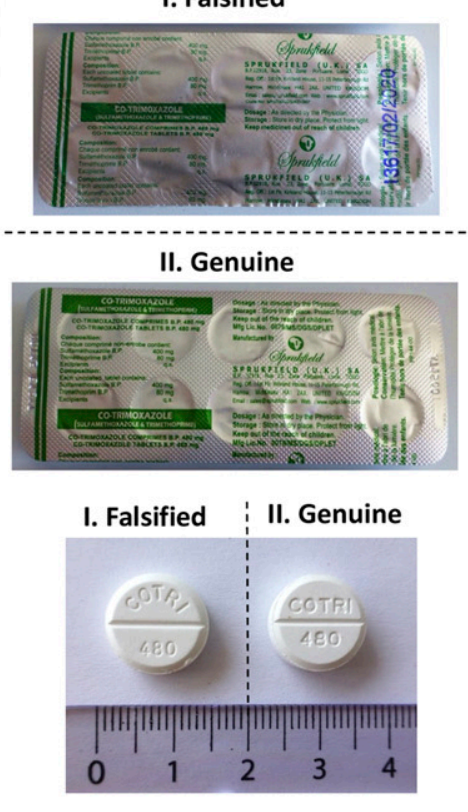
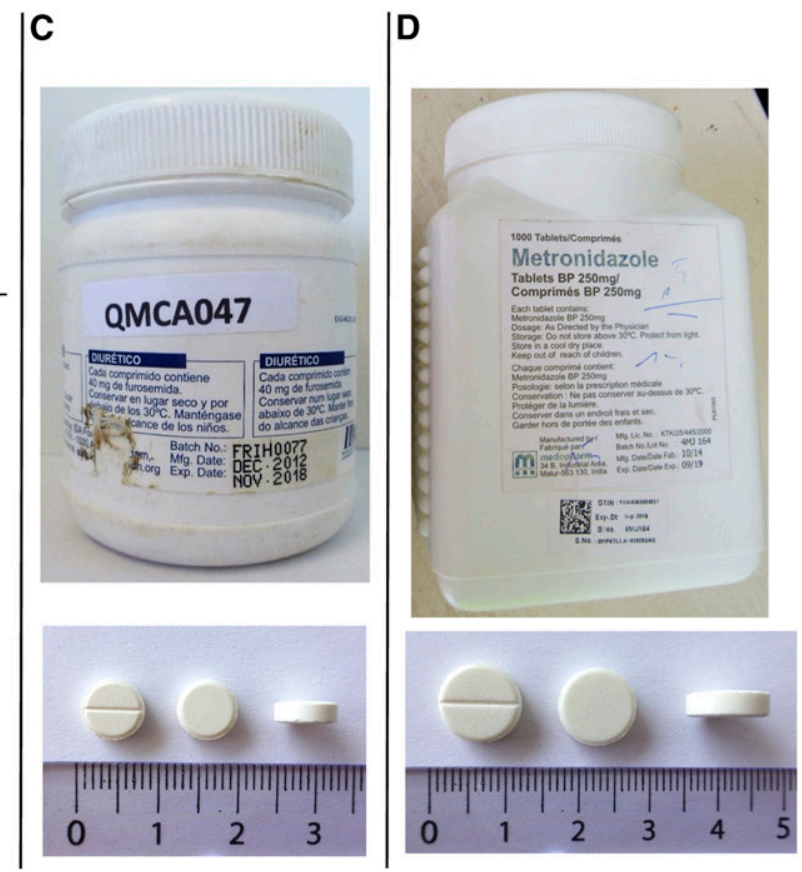

FIGURE 4. Photos of falsified medicines identified by authenticity inquiries. (A) Falsified "Amoxicillin $500 \mathrm{mg}+$ Clavulanic acid $125 \mathrm{mg}$ BP" tablets. Batch number, shelf life, blister length (shown in Supplemental Figure S2), and further labeling details do not match those of the genuine product, according to the stated manufacturer and distributor. (B) Falsified (I) and genuine (II) "CO-TRIMOXAZOLE (sulfamethoxazole \& trimethoprim)" tablets. The falsified sample shows an incorrect batch number. Batch number and expiry date are printed rather than being embossed onto the blister; blister length, artwork, and color of the blister, as well as the embossing of the tablets, do not match those of the genuine product. The falsified product had been sold without secondary packaging. (C) One of the four falsified "Furosemide 40 mg BP" samples with a manipulated expiry date ("Nov. 2018", instead of the genuine expiry date "Nov. 2015"). (D) Falsified "Metronidazole Tablets BP 250 mg" also showing a manipulated expiry date ("09/19" instead of the genuine expiry date "09/17"). This figure appears in color at www.ajtmh.org.

Therefore, these four samples may have been genuine products in their genuine packages, but their expiry dates (and in two samples also their manufacturing dates) apparently had been manipulated with deliberate/fraudulent intent, turning them into falsified products.

Another sample (Figure 4D) had been collected in a government health facility in Nord Kivu, the DR Congo, and was labeled as "Metronidazole Tablets BP 250 mg," stated manufacturer: Medopharm (Malur, India). On inquiry, the stated manufacturer confirmed that a product with the same name, batch number, manufacturing date, and artwork had been manufactured by them, but that the expiry date of the sample ("09/19") did not match with that of the genuine product ("09/ 17 "). The sample showed a second, small label with a twodimensional bar code (Figure 4D), and the manufacturer reported that this encoded the correct expiry date (September $30,2017)$, in contrast to the expiry date written in plain text on the same label ("Sep-2019"). This indicates that, on an otherwise genuine package, the labeling may have been modified to extend the expiry date by 2 years.

The WHO Rapid Alert System and the national drug regulatory authorities were informed about these falsifications.

\section{DISCUSSION}

A key aim of the present article is to contribute to an improved comparability of prevalence data of SF medicines reported in scientific studies. Figure 2 strikingly illustrates that the calculated prevalence rates of OOS medicines are extremely dependent on the choice of the tolerance limits used in the respective study. From the analytical data obtained for our "real-world" sample collection of medicines from three LMICs, the calculated rate of OOS medicine is as low as 3.3\% or as high as $35.0 \%$, simply by choosing different tolerance limits to distinguish "in-specification" and "out-of-specification" results for the content of the API. Notably, these different tolerance limits have been used in recent, large medicine quality studies, ${ }^{7,17,28}$ and the results of these studies have been included into recent systematic reviews, which then calculated overall prevalence rates of SF medicines by aggregating numbers of "poor-quality" medicines reported in the included studies., 3

As correctly stated by McManus and Naughton, ${ }^{5}$ the results of systematic reviews are only as reliable as the original data to which they refer. If the arbitrary choice of tolerance limits allows the rate of OOS medicines in the same study to vary by a factor of more than 10 (as shown in Figure 2), the results of systematic reviews obviously become unreliable, and, for example, comparisons of the rates of OOS medicines between different time periods, as attempted in the systematic review by McManus and Naughton, ${ }^{5}$ may become meaningless. Given the important public health and socioeconomic effects of SF medicines, and the importance of reliable and comparable prevalence data for such medicines, a harmonization of the tolerance limits used to distinguish compliant from OOS products in different medicine quality studies is urgently called for, as also concluded from a recent study by the Brazilian Health Regulatory Agency. ${ }^{29}$ Figure 2 clearly demonstrates that arbitrary tolerance limits, such as $85-115 \%$ or $95-105 \%$, should be avoided, and pharmacopoeial tolerance limits should be used instead. Indeed, the group of $\mathrm{H}$. Kaur who had used the $85-115 \%$ limits in their cited studies ${ }^{7,28}$ decided to use USP tolerance limits in their later studies. ${ }^{30}$ The BP uses stricter tolerance limits than USP and $\mathrm{Ph}$. Int. for many medicines. Both BP and USP state in their "General Notices" that these tolerance limits allow for analytical 
errors, for unavoidable variations in manufacturing, and for deterioration to an acceptable extent. ${ }^{31,32}$ To our knowledge, there is no published information why these tolerance limits differ between different pharmacopoeias.

In our real-world sample collection, the use of BP specifications for assay results led to an approximately 2 -fold higher number of OOS medicines than the use of USP or Ph. Int. specifications. As mentioned in the Methods section, $\mathrm{WHO}$ guidelines ${ }^{14}$ recommend that in one medicine quality study, all samples which contain the same API in the same dosage form should be tested using the same methods and specifications. In our studies, many products were labeled as manufactured according to USP specifications, and it would obviously be inappropriate to evaluate these samples according to the narrower tolerance limits of BP. Therefore, we suggest to use USP or the similar Ph. Int. specifications, rather than BP specifications, in medicine quality studies involving more than one country. Obviously, it is desirable that the specifications of different pharmacopoeias are harmonized as soon as possible, to create global and uniform public standards for medicines. ${ }^{33-36}$

For a number of medicines, no pharmacopoeial monograph exists, ${ }^{34}$ and in these cases, the tolerance limits for medicines of the same therapeutic class which are included in the USP or $\mathrm{Ph}$. Int. may be used. For assay, this would result in the use of tolerance limits of $90-110 \%$ in many cases. ${ }^{29}$ Special considerations may be required for medicines with a narrow therapeutic index. The tolerance limits stated in the in-house specifications of the respective manufacturers may also be considered. However, as mentioned earlier, in one study, all samples which contain the same API in the same dosage form should be tested using the same specifications. ${ }^{14}$

The authoritative review by the $\mathrm{WHO}^{2}$ stated the problem that most published medicine quality studies only report a pass or fail result for the medicines samples, rather than reporting the actual percentage of APIs detected (or dissolved). As illustrated in Figure 1, much more information can be gained if actual percentages for API content, and also dissolution, ${ }^{15}$ are shown for each sample, and this also allows much more meaningful comparisons between different studies.

Several previous medicine quality studies published by the $\mathrm{WHO}^{37,38}$ have differentiated "substandard" samples into samples showing "moderate deviations" and "extreme deviations" from pharmacopoeial specifications, with extreme deviations defined as the content of APIs deviating by more than $20 \%$ from the declared content, and/or the average dissolution value of tested units of solid oral formulations falling below the pharmacopoeial $\mathrm{Q}$ value minus $25 \%$. Although this classification method is rapidly and universally applicable, it has the drawback that it does not consider the different health risks of dosage deviations from medicines with different therapeutic indices.

Based on the MEDQUARG guidelines by Newton et al., ${ }^{13}$ Almuazini et al. ${ }^{4}$ have developed a 12-point checklist for the rating of the quality of published medicine quality studies, and this has been used in several subsequent systematic reviews. ${ }^{2,3,5,39}$ We suggest that, in future, this checklist is expanded by the following two criteria:

1. "Actual percentages are shown for assay and (if applicable) for dissolution values of each sample."

2. "Preferentially, USP or Ph. Int. tolerance limits are used to distinguish in-specification from OOS medicine samples; the choice of other tolerance limits should be justified."
Within the present study, we carried out an authenticity inquiry by contacting the stated manufacturers and distributors of the same medicines. As noted by the $\mathrm{WHO},{ }^{2}$ only few studies have reported the authentication of medicine samples using this method, and we therefore documented the procedures and results of this exercise in detail (see Methods and Results sections). Notably, although packaging and chemical analyses had identified three $(0.5 \%)$ of 601 samples as falsified, authentication by manufactures and distributors increased this number to $10(1.7 \%)$. This clearly shows that authentication is a powerful tool for medicine quality studies. However, the workload for such inquiries is high, both for the researchers and for the manufacturers and distributors. Furthermore, response rates are far from complete. Four recent studies by Kimura and coworkers reported that authenticity responses were received from the manufacturers for $0 \%, 8.7 \%, 9.5 \%$, and $28.8 \%$ of the investigated samples, respectively. ${ }^{40-43}$ In comparison, the response rate of $49.5 \%$ obtained in the present study is reasonably high, despite interference by the COVID-19 pandemic. Higher response rates were reported from two earlier studies of the Kimura group. ${ }^{44,45}$

Although the statement of the manufacturer and distributor is an important piece of evidence in the identification of falsified medicines, it cannot be taken for granted that this statement reflects the truth in all cases. If a manufacturer doubts whether his product passed quality testing in the respective study, he may be tempted to deny its authenticity rather than risking a published report that his genuine product was found to be substandard.

Notably, all seven falsified samples identified through the authenticity inquiry passed chemical analysis for assay and dissolution without any conspicuous deviations from USP specifications. Similar observations have been reported by Kimura and coworkers. ${ }^{44,45}$ However, this does not mean that such falsifications are of no concern for patient safety. A medicine which misrepresents its source, or which carries an illegally modified expiry date, must be considered dangerous, as it may fail specifications which had not been tested in our studies, such as limits for chemical and microbial contaminations.

Some researchers have suggested that in medicine quality studies, it may be sufficient to report a single prevalence rate for "substandard and falsified medicines" combined together. In contrast to this suggestion, however, the WHO has defined "substandard" and "falsified" medicines as different categories. ${ }^{2,10}$ We believe this was a prudent decision, because very different interventions are required to respond to the problem of falsified medicines, as compared with the problem of substandard medicines. Informed decision-making requires knowledge of both prevalence rates.

In a medicine quality study, the number of "out-of-specification" medicines can be determined by laboratory analysis. "Substandard" medicines are those OOS medicines for which no evidence is available that they deliberately/fraudulently misrepresent their identity, composition, or source. By contrast, "falsified" medicines are those for which evidence of such deceit is available, irrespective of whether their analytical results are "in specification" or "out of specification." The present study has shown examples for all these categories. Of 601 medicine samples which we collected in Cameroon, the DR Congo, and Malawi, 99 (16.5\%) have thereby been identified as substandard based on USP specifications for assay 
and dissolution (or Ph. Int. specifications in case of misoprostol tablets), and $10(1.7 \%$ ) have been identified as falsified.

These categorizations cannot be free of ambiguity in scientific practice. ${ }^{12}$ For example, even when a product has passed visual inspection, chemical analysis, and authenticity inquiries, it may still be falsified if labeling and chemical composition of the authentic product have been imitated very closely. Furthermore, defining criteria by which deliberate/ fraudulent intent should be proven will always be a controversial issue. ${ }^{12}$ And in case of OOS medicines, definitive proof of absence of such intent is virtually impossible.

To harmonize the reporting of "falsified" medicine samples, also in the absence of authoritative legal proof of deliberate intent or fraud, it may be considered that medicine quality studies by academia or NGOs use the term "falsified" in the following cases:

1. A responsible national or international authority has declared that the medicine is falsified, based on the $\mathrm{WHO}$ definitions. $^{10,46}$

2. The stated manufacturer has declared that the medicine is falsified, or that its expiry date or other details of the labeling have been illegally manipulated. ${ }^{46}$

3. Packaging analysis gives conclusive evidence for falsification, for example, the stated manufacturer does not exist.

4. The medicine contains no APIs, or an incorrect API instead of the stated one. (In the very rare event that this mistake resulted from non-intentional errors in both production and quality control, the medicine has to be classified as "substandard".)

In addition, it may be considered to use the term "probably falsified" for medicines which contain less than $50 \%$ of the stated amount of the API AND for which there is no evidence that the low content is due to degradation (evidenced usually by the presence of decomposition products). An example for such a medicine is given by chloroquine tablets which we recently found in Cameroon and which contained only $21.7 \%$ of the declared amount of the API. ${ }^{47,48}$ On the other hand, we identified a brand of misoprostol tablets in Malawi which contained only $13.1 \%$ of the stated amount of the API, ${ }^{16}$ but HPLC analysis clearly showed the presence of decomposition products of misoprostol, and therefore, this product was considered as "extremely substandard" rather than "probably falsified". ${ }^{16}$

We realize that different opinions exist in the scientific community and within the stakeholders regarding the best possible categorization of SF medicines in scientific studies. We hope that the facts and suggestions presented in this article may help to stimulate discussions in search of a consensus, and contribute to the urgently needed harmonization of the methodology and reporting of future medicine quality studies.

Limitations of this study. Comparability of the results of medicine quality studies also depends on other factors beyond the scope of the present article, such as sampling design (e.g., random or convenience sampling, overt or mystery shopping, inclusion or exclusion of informal vendors, and choice of included medicines), methods of chemical analysis, and choice of tests included into the investigation (e.g., assay, dissolution, content uniformity, related compounds, etc.). This article focuses on the classification of medicines as "substandard" and "falsified," but does not reflect on the classification as "unregistered/unlicensed" which was also defined by the World Health Assembly of $2017 . .^{10,49}$ Also, this article focuses only on the APIs, and did not consider quality issues related to excipients.

Received December 21, 2020. Accepted for publication February 5, 2021.

Published online March 31, 2021.

Note: Supplemental tables and figures appear at www.ajtmh.org

Acknowledgments: We are grateful to Richard Neci (Ecumenical Pharmaceutical Network, Nairobi, Kenya), Georges Mutombo (Le Dépôt Central Médico-Pharmaceutique de la 8e CEPAC [DCMP], Bukavu, the Democratic Republic of the Congo), Edward Ngah Ndze and Tambo Cletus (Cameroon Baptist Convention [CBC], Central Pharmacy, Mutengene, Cameroon), Fidelis Nyaah and Manyi Pattinora (Presbyterian Church in Cameroon [PCC], Central Pharmacy, Limbe, Cameroon), Felix Khuluza (Pharmacy Department, College of Medicine, University of Malawi), Simon Schäfermann, Emmanuel Wemakor, Irina Helmle, Dorothee Wistuba and Harald Gross (Eberhard Karls University Tuebingen, Germany), and Christine HäfeleAbah (German Institute for Medical Mission [Difaem], Tuebingen, Germany) for their contributions to the cited medicine quality studies carried out in the DR Congo, in Cameroon, and in Malawi.

Financial support: This study was funded by the Eberhard Karls University, Tuebingen.

Authors' addresses: Cathrin Hauk, Nhomsai Hagen, and Lutz Heide, Pharmaceutical Institute, Eberhard Karls University, Tuebingen, Germany, E-mails: cathrin.hauk@uni-tuebingen.de, nhomsai.hagen@unituebingen.de, and heide@uni-tuebingen.de.

This is an open-access article distributed under the terms of the Creative Commons Attribution (CC-BY) License, which permits unrestricted use, distribution, and reproduction in any medium, provided the original author and source are credited.

\section{REFERENCES}

1. Nayyar GM, Attaran A, Clark JP, Culzoni MJ, Fernandez FM, Herrington JE, Kendall M, Newton PN, Breman JG, 2015. Responding to the pandemic of falsified medicines. Am J Trop Med Hyg 92 (Suppl 6): 113-118.

2. World Health Organization, 2017. A Study on the Public Health and Socioeconomic Impact of Substandard and Falsified Medical Products. Available at: http://www.who.int/medicines/regulation/ ssffc/publications/se-study-sf/en/. Accessed February 4, 2021.

3. Ozawa S, Evans DR, Bessias S, Haynie DG, Yemeke TT, Laing SK, Herrington JE, 2018. Prevalence and estimated economic burden of substandard and falsified medicines in low- and middle-income countries: a systematic review and meta-analysis. JAMA Netw Open 1: e181662.

4. Almuzaini T, Choonara I, Sammons H, 2013. Substandard and counterfeit medicines: a systematic review of the literature. BMJ Open 3: e002923.

5. McManus D, Naughton BD, 2020. A systematic review of substandard, falsified, unlicensed and unregistered medicine sampling studies: a focus on context, prevalence, and quality. BMJ Glob Health 5: e002393.

6. Nayyar GM, Breman JG, Newton PN, Herrington J, 2012. Poorquality antimalarial drugs in southeast Asia and sub-Saharan Africa. Lancet Infect Dis 12: 488-496.

7. Kaur Het al., 2016. Fake anti-malarials: start with the facts. Malar $J$ 15: 86.

8. Khuluza F, Kigera S, Heide L, 2017. Low prevalence of substandard and falsified antimalarial and antibiotic medicines in public and faith-based health facilities of southern Malawi. Am J Trop Med Hyg 96: 1124-1135.

9. Chikowe I, Osei-Safo D, Harrison JJ, Konadu DY, Addae-Mensah I, 2015. Post-marketing surveillance of anti-malarial medicines used in Malawi. Malar J 14: 127.

10. World Health Organization, 2017. Who Member State Mechanism on Substandard/Spurious/Falsely-Labelled/Falsified/Counterfeit (SSFFC) Medical Products. Working Definitions. Available at: 
https://www.who.int/medicines/regulation/ssffc/definitions/en/. Accessed February 4, 2021.

11. U.S. Department of Health and Human Services, Food and Drug Administration, Center for Drug Evaluation and Research (CDER), 2006. Guidance for Industry. Investigating Out-of-Specification (OOS) Test Results for Pharmaceutical Production. Available at: https:// www.fda.gov/media/71001/download.

12. Olliaro E, Olliaro $P$, Ho CWL, Ravinetto R, 2020. Legal uncertainty-the gray area around substandard medicines: where public health meets law. Am J Trop Med Hyg 102: 262-267.

13. Newton PN et al., 2009. Guidelines for field surveys of the quality of medicines: a proposal. PLoS Med 6: e52.

14. WHO Expert Committee on Specifications for Pharmaceutical Preparations, 2016. Guidelines on the Conduct of Surveys of the Quality of Medicines. WHO Technical Report Series No. 996. Available at: https://www.who.int/medicines/publications/ pharmprep/trs_996/en/. Accessed February 4, 2021.

15. Schafermann S et al., 2020. Substandard and falsified antibiotics and medicines against noncommunicable diseases in western Cameroon and northeastern Democratic Republic of Congo. Am J Trop Med Hyg 103: 894-908.

16. Hagen N, Khuluza F, Heide L, 2020. Quality, availability and storage conditions of oxytocin and misoprostol in Malawi. BMC Preg Childbirth 20: 184.

17. Antignac $M$ et al., 2017. Fighting fake medicines: first quality evaluation of cardiac drugs in Africa. Int J Cardiol 243: 523-528.

18. FDA, 2017. Acceptability of Standards from Altemative Compendia (BP/ $E P / J P)$. Available at: https://www.fda.gov/media/72412/download.

19. Ammar HO, Khalil RM, 1996. Discrepancy among dissolution rates of commercial tablets as a function of dissolution method. Part 6: rifampicin. Pharmazie 51: 165-168.

20. Panchagnula R, Bhardwaj V, 2008. Effect of amorphous content on dissolution characteristics of rifampicin. Drug Dev Ind Pharm 34: 642-649.

21. Becker C, Dressman JB, Junginger HE, Kopp S, Midha KK, Shah VP, Stavchansky S, Barends DM, 2009. Biowaiver monographs for immediate release solid oral dosage forms: rifampicin. J Pharm Sci 98: 2252-2267.

22. Hauk $C$, Schäfermann $S$, Martus $P$, Muzafarova $N$, Babaley $M$, Waning B, Heide L, 2020. Quality assurance in anti-tuberculosis drug procurement by the Stop TB Partnership-Global Drug Facility: procedures, costs, time requirements, and comparison of assay and dissolution results by manufacturers and by external analysis. PLoS One 15: e0243428.

23. Schiavetti B, Wynendaele E, Melotte V, Van der Elst J, De Spiegeleer B, Ravinetto R, 2020. A simplified checklist for the visual inspection of finished pharmaceutical products: a way to empower frontline health workers in the fight against poorquality medicines. J Pharm Policy Pract 13: 9.

24. World Health Professions Alliance, 2011.BeAware: Tool for Visual Inspection of Medicines. Available: https://www.whpa.org/ sites/default/files/2018-12/Toolkit_BeAware_Inspection.pdf, 4. Accessed February 4, 2021.

25. World Health Organization, 2017. Medical Product Alert No 4/2017. Falsified Penicillin V Circulating in Cameroon. Ref. RHT/SAVIAlert_ n4.2017. Available at: https://www.who.int/medicines/publications/ drugalerts/drug_alert4-2017/en/. Accessed February 4, 2021.

26. Hagen N, Bizimana T, Kayumba PC, Khuluza F, Heide L, 2020. Stability of oxytocin preparations in Malawi and Rwanda: stabilizing effect of chlorobutanol. Am J Trop Med Hyg 103: 2129-2141.

27. World Health Organization, 2018. Medical product alert $N^{\circ} 2 /$ 2018. Falsified "Augmentin" circulating in Cameroon. Ref. RHT/ SAV/Alert n2.2018. Available at: https://www.who.int/ medicines/publications/drugalerts/drug_alert2-2018/en/. Accessed December 14, 2020.

28. Kaur $\mathrm{H}$ et al., 2015. Quality of artemisinin-based combination formulations for malaria treatment: prevalence and risk factors for poor quality medicines in public facilities and private sector drug outlets in Enugu, Nigeria. PLoS One 10: e0125577.

29. Neves EO, de Sales PM, Silveira D, 2020. Pharmacopeial specifications and analytical data from post-marketing quality sampling and testing programs: a perspective beyond out-ofspecification results. J Pharm Biomed Anal 178: 112935.

30. Redfern J, Kaur H, Adedoyin RA, Ofori S, Anchala R, Vamadevan AS, De Andrade L, Zelaya J, Balabanova D, Sani MU, 2019.
Equivalence in active pharmaceutical ingredient of generic antihypertensive medicines available in Nigeria (EQUIMEDS): a case for further surveillance. Glob Heart 14: 327-333.

31. USP 42 - NF 37, 2019. The United States Pharmacopeia and National Formulary 2019. Rockville, MD: United States Pharmacopeial Convention.

32. British Pharmacopoiea (BP), 2020. London, United Kingdom: British Pharmacopoiea Commission.

33. World Health Organization, 2020. White Paper for the WHO International Meeting of World Pharmacopoeias - Value of Pharmacopoeial Standards for Access to Quality Medicines. Available at: https://www.who.int/docs/default-source/medicines/normsand-standards/value-of-pharm-stds-updated-final-oct-2020.pdf? sfursn=2d4e5052_4. Accessed February 4, 2021.

34. Roth L, Adler M, Jain T, Bempong D, 2018. Monographs for medicines on WHO's model list of essential medicines. Bull World Health Organ 96: 378-385.

35. EDQM Council of Europe. The Need for International Harmonisation. Available at: https://www.edqm.eu/en/internationalharmonisation. Accessed February 4, 2021.

36. World Health Organization. Norms and Standards for Pharmaceuticals. Available at: https://www.who.int/teams/health-productand-policy-standards/standards-and-specifications/norms-andstandards-for-pharmaceuticals/. Accessed February 4, 2021.

37. World Health Organization, 2015. Survey of the Quality of Medicines Identified by the United Nations Commission on LifeSaving Commodities for Women and Children. Availabel at: https://apps.who.int/iris/handle/10665/255550. Accessed February $4,2021$.

38. World Health Organization, 2011. Survey of the Quality of Selected Antimalarial Medicines Circulating in Six Countries of Sub-Saharan Africa. Available at: http://www.who.int/medicines/publications/ WHO_QAMSA_report.pdf. Accessed February 4, 2021.

39. Torloni MR, Bonet M, Betran AP, Ribeiro-do-Valle CC, Widmer M, 2020. Quality of medicines for life-threatening pregnancy complications in low- and middle-income countries: a systematic review. PLoS One 15: e0236060.

40. Rahman MS, Yoshida N, Sugiura S, Tsuboi H, Keila T, Kiet HB, Zin T, Tanimoto T, Kimura K, 2018. Quality of omeprazole purchased via the Internet and personally imported into Japan: comparison with products sampled in other Asian countries. Trop Med Int Health 23: 263-269.

41. Islam MR et al., 2018. An investigation into the quality of medicines in Yangon, Myanmar. Pharmacy (Basel) 6: 96.

42. Rahman MS et al., 2019. A cross-sectional investigation of the quality of selected medicines for noncommunicable diseases in private community drug outlets in Cambodia during 2011-2013. Am J Trop Med Hyg 101: 1018-1026.

43. Sakuda M, Yoshida N, Takaoka T, Sanada T, Rahman MS, Tanimoto T, Zin T, Kimura K, Tsuboi H, 2020. Substandard and falsified medicines in Myanmar. Pharmacy (Basel) 8: 45.

44. AkaE, Legris C, Tanimoto T, Matsushita R, Kimura K, 2005. Counterfeit medicine detection by authenticity investigation. A pilot study in the street market of Côte d'Ivoire. Jpn J Soc Pharm 24: 7-16.

45. Khan MH, Okumura J, Sovannarith T, Nivanna N, Akazawa M, Kimura K, 2010. Prevalence of counterfeit anthelminthic medicines: a cross-sectional survey in Cambodia. Trop Med Int Health 15: 639-644.

46. World Health Organization, 2017. WHO Global Surveillance and Monitoring System for Substandard and Falsified Medical Products. Available at: http://www.who.int/medicines/regulation/ssffc/ publications/gsms-report-sf/en/. Accessed February 4, 2021.

47. Gnegel G, Hauk C, Neci R, Mutombo G, Nyaah F, Wistuba D, Hafele-Abah C, Heide L, 2020. Identification of falsified chloroquine tablets in Africa at the time of the COVID-19 pandemic. Am J Trop Med Hyg 103: 73-76.

48. World Health Organization, 2020. Medical Product Alert No4/ 2020. Falsified Chloroquine Products Circulating in the WHO Region of Africa. Available at: https://www.who.int/newsroom/detail/09-04-2020-medical-product-alert-n4-2020. Accessed February 4, 2021.

49. World Health Organization, 2017. Seventieth World Health Assembly Update. Available at: http://www.who.int/en/news-room/detail/ 29-05-2017-seventieth-world-health-assembly-update-29may-2017. Accessed February 4, 2021. 